\title{
Detecting polymeric nanoparticles with Coherent Anti-Stokes Raman scattering microscopy in tissues exhibiting fixative-induced autofluorescence
}

\author{
N. L. Garrett*a , L. Godfrey ${ }^{\mathrm{b}}$, A. Lalatsa ${ }^{\mathrm{c}}$, D. R. Serrano ${ }^{\mathrm{d}}$, I. F. Uchegbu ${ }^{\mathrm{b}}$, A. Schatzlein ${ }^{\mathrm{b}}$, J. Moger $^{\mathrm{a}}$ \\ ${ }^{a}$ School of Physics, University of Exeter, Exeter, Devon, EX4 4QL UNITED KINGDOM UCL \\ ${ }^{\mathrm{b}}$ School of Pharmacy, 29-39 Brunswick Square, London WC1N 1AX, UK \\ ${ }^{\mathrm{c}}$ Pharmacy and Biomedical Sciences, University of Portsmouth, White Swan Road Portsmouth PO1 \\ 2DT \\ ${ }^{\mathrm{d}}$ Departmento de Farmacia y Tecnologia Farmaceutica, Facultad de Farmacia, Universidad \\ Complutense de Madrid, Plaza Ramon y Cajal s/n, Madrid, 28040, Spain
}

\begin{abstract}
Recent advances in pharmaceutical nanotechnology have enabled the development of nano-particulate medicines with enhanced drug performance. Although the fate of these nano-particles can be macroscopically tracked in the body (e.g. using radio-labeling techniques), there is little information about the sub-cellular scale mechanistic processes underlying the particle-tissue interactions, or how these interactions may correlate with pharmaceutical efficacy. To rationally engineer these nano-particles and thus optimize their performance, these mechanistic interactions must be fully understood.

Coherent Anti-Stokes Raman scattering (CARS) microscopy provides a label-free means for visualizing biological samples, but can suffer from a strong non-resonant background in samples that are prepared using aldehyde-based fixatives. We demonstrate how formalin fixative affects the detection of polymeric nanoparticles within kidneys following oral administration using CARS microscopy, compared with samples that were snap-frozen. These findings have implications for clinical applications of CARS for probing nanoparticle distribution in tissue biopsies.
\end{abstract}

Keywords: CARS, Raman, nanoparticle, drug delivery, microscopy, imaging, autofluorescence

\section{INTRODUCTION}

Nanoparticle drug delivery is an area of medical research that is rapidly expanding to provide novel methods for transporting drugs to target organs. Recent advances in polymer formulations using biocompatible quaternary ammonium palmitoyl glycol chitosan (GCPQ) have resulted in nanoparticles capable of encapsulating hydrophobic drugs for enhanced delivery via IV and oral dosing routes ${ }^{1-3}$. Determining the toxicity of these nanoparticles is obviously of great importance and the mechanisms by which the nanoparticles interact with the body at the cellular level must be understood in order to intelligently engineer novel nano-medicines and better understand their modes of action.

Nanoparticle uptake mechanisms at the cellular scale are currently determined using techniques such as Confocal Laser Scanning Microscopy (CLSM), which rely on the addition of extrinsic labels such as fluorophores. These fluorophores introduce complications for the analysis of the nanoparticle kinetics, since the labels themselves can alter the biological properties of the nanoparticle. There are additional complications with CLSM such as: photobleaching of the sample and frequently toxic labels. Radio-isotope labelling is an alternative technique, whereby substitutions are made of one or more isotopes of an element within a molecule. For instance, hydrogen atoms can be replaced with deuterium in molecules of interest as it enables detection of the molecule without adversely affecting the system's biochemical interactions within an organism ${ }^{4}$.

*n.1.garrett@exeter.ac.uk; phone+44(0) 1392724155

Multiphoton Microscopy in the Biomedical Sciences XV, edited by Ammasi Periasamy, Peter T. C. So, Karsten König, Proc. of SPIE Vol. 9329, 932922 - (C) 2015 SPIE · CCC code: 1605-7422/15/\$18 · doi: 10.1117/12.2079365 
This method of deuterium labelling has been used in NMR studies of humans to determine the PK and PD of compounds ${ }^{5}$. Deuterated bonds may also be distinguished against a biological background using spectroscopic analysis ${ }^{6}$. In Raman spectroscopy, the $\mathrm{CH}_{2}$ stretching mode is located at $2840 \mathrm{~cm}^{-1}$ whereas when hydrogen is replaced with deuterium, the $\mathrm{CD}_{2}$ stretching mode is located at $2100 \mathrm{~cm}^{-1}$. Therefore it is possible to detect a deuterated molecule of interest by probing the sample at $2100 \mathrm{~cm}^{-1}$, which lies within what is referred to as the 'silent region' in biological samples.

With this technique, Raman microscopy studies of human cells have used deuterium-labelled proteins without altering the cells' chemical environment ${ }^{7}$. In Raman spectroscopy, the intensity of laser light scattered from a sample is measured in units of wave numbers $\left(\mathrm{cm}^{-1}\right)$ as a function of the energy shift (Raman shift) with respect to the incident beam. The scattered light's energy is shifted relative to the incident beam as a result of interactions with the sample's Raman-active chemical bonds; the Raman spectrum contains narrow peaks corresponding with particular chemical bonds, therefore giving a unique 'fingerprint'. The number of Raman-active bonds within the sample is directly proportional to intensity of the Raman scattered light, thus allowing straightforward quantitative analysis from the detected signal.

Spontaneous Raman scattering would be a potentially attractive method for obtaining chemically selective information from biological specimens, were it not such a prohibitively weak process. This is illustrated by comparing the typical Raman cross-sections per molecule for Raman scattering, which range from 10-25 to 10-30 $\mathrm{cm}^{2}$, with typical crosssections for fluorescence, which range from 10-16 to $10-17 \mathrm{~cm}^{2}$. Although increasing the incident laser intensity would theoretically improve the signal-to-noise ratio for spontaneous Raman scattering, in practice this would rapidly cause photo-thermal damage to biological specimens. The limitations of spontaneous Raman scattering can be overcome using the non-linear process, coherent anti-Stokes Raman scattering (CARS). Advancements in ultrafast laser technology have allowed CARS microscopy to be developed as a tool for label-free imaging with chemical specificity by probing the vibrational structure of molecules within the sample volume ${ }^{8,9}$. CARS is a four-wave mixing process in which a pump beam of frequency $\mathrm{w}_{\mathrm{p}}$ and a Stokes beam, $\mathrm{w}_{\mathrm{s}}$, interact within a sample. By tuning the beat frequency $\left(\mathrm{w}_{\mathrm{p}}-\mathrm{w}_{\mathrm{s}}\right)$ to match a Raman active vibrational mode, the excitation fields coherently drive the sample molecules resulting in the generation of a strong anti-Stokes signal at $\left(2 \mathrm{w}_{\mathrm{p}}-\mathrm{w}_{\mathrm{s}}\right)$. CARS microscopy has many advantages over conventional imaging including: up to several hundred micron depth penetration into biological tissue; intrinsic optical sectioning and high spatial resolution; label-free chemically specific contrast. Of the many applications of CARS microscopy, numerous investigations have focused on directly visualising the trafficking of particles, including that of lipid droplets ${ }^{10}$, gold nanoshells ${ }^{11}$, metal oxides ${ }^{12}$ and nano-medicines ${ }^{13}$.

Although CARS is a powerful chemically-specific imaging technique, it is not background-free; in addition to the resonant contribution to the CARS signal, there is also an inherent non-resonant component ${ }^{9,14-16}$. In biological specimens, this non-resonant background manifests itself as a substantial CARS signal arising predominantly from the sample's bulk water ${ }^{15,16}$. Since the anti-Stokes signal is blue-shifted with respect to the incident near infrared excitation wavelengths of the pump and Stokes beams, there is diminished fluorescence contribution to the detected CARS signal. However, the autofluorescence of the sample is not zero; although it is true that at excitation wavelengths longer than $800 \mathrm{~nm}$ there is little two-photon pyridine nucleotide $(\mathrm{NAD}(\mathrm{P}) \mathrm{H})$ fluorescence, the two-photon excitation spectra of lipoamide dehydrogenase (LipDh) and flavin adenine dinucleotide (FAD) actually extend to $1000 \mathrm{~nm}$ and have broad peaks around $900 \mathrm{~nm}$ which allow for excitation of flavoproteins, the emission from which can be detected even up to $650 \mathrm{~nm}{ }^{17}$. Therefore, even when the often-selected CARS excitation wavelengths of $924 \mathrm{~nm}$ and $1254 \mathrm{~nm}$ are used to excite contrast from the $\mathrm{CH}_{2}$ stretch, when detecting the anti-Stokes shifted signal at around $731 \mathrm{~nm}$ there can still be some background autofluorescence contribution (the magnitude of this will partly depend on the bandwidth of the filter used to isolate the anti-Stokes signal).

It is clear that sample autofluorescence has the potential to limit the sensitivity of CARS when detecting sub-voxel sized nanoparticles against biological backgrounds. The process by which the sample has been processed can affect the levels of fluorescence. For instance, samples that have been fixed using aldehydes (e.g. formaldehyde, gluteraldehyde etc.) tend to exhibit aldehyde-induced fluorescence arising from Schiff bases which form during the reaction of aldehydes and the tissues' proteins, thus creating epsilon amino groups ${ }^{18}$. Additionally, some alcohol-based fixation techniques can strip away lipids from the sample, the effects of which have been discussed for imaging brain tissues with CARS $^{19}$. Samples obtained during tissue biopsies in the operating theatre may be snap-frozen or fixed depending on the protocol in use at the time; therefore, there is a need to determine just what effect, if any, sample preparation has 
on the ability to detect nanoparticles within tissues using CARS. If CARS microscopy is ever to be used in a clinical setting to determine nanoparticle delivery to human organs (e.g. to assess distribution and toxicity), it is crucial that the effects of sample preparation are determined beforehand.

The ideal drug delivery system would transport the drug to the target site (e.g. the brain) whilst simultaneously avoiding build-up of drug within organs to which the drug is toxic (e.g. the kidneys). The ability to visualize nanoparticles within organs such as the kidneys to confirm that there is no significant cellular uptake of potentially toxic drugs is therefore of critical importance for development of new nanomedicines. In this study we investigated the effect of sample preparation on the ability of CARS microscopy to pin-point deuterated GCPQ nanoparticles in kidneys that were either fixed using formaldehyde-based fixative or snap frozen using liquid nitrogen.

\section{METHODS}

\subsection{Spontaneous Raman scattering spectroscopy}

Raman spectra were acquired using a Renishaw RM1000 Raman microscope (Renishaw, Wotton-Under-Edge, UK) equipped with a 1200-line/mm grating providing a spectral resolution of $1 \mathrm{~cm}^{-1}$ and a diode laser providing excitation at $785 \mathrm{~nm}$ with up to $300 \mathrm{~mW}$ power. The system was calibrated using the Raman band of a silicon wafer at $520 \mathrm{~cm}^{-1}$. Spectral data was acquired using Renishaw v.1.2 WiRE software.

\subsection{Coherent Anti-Stokes Raman scattering microscopy}

Coherent Anti-Stokes Raman Scattering (CARS) microscopy was performed using a custom built imaging system based on a modified commercial confocal laser scanning microscopy and a synchronised dual-wavelength picosecond laser source, as shown in the schematic diagram in figure 1. Laser excitation was provided by an optical parametric oscillator (OPO) (Levante Emerald, APE, Berlin) pumped with a frequency doubled Nd:Vandium picosecond oscillator (High-Q Laser Production $\mathrm{GmbH}$ ). The pump laser generated a $6 \mathrm{ps}, 76 \mathrm{MHz}$ pulse train at $532 \mathrm{~nm}$ with adjustable output power up to $10 \mathrm{~W}$. The OPO produced collinear signal and idler beams with perfect temporal overlap and provided continuous tuning over a range of wavelengths. The signal beam was used as the pump, ranging from 670 to $980 \mathrm{~nm}$ and the pump laser was used as the Stokes beam at $1064 \mathrm{~nm}$. The maximum combined output power of the signal and idler was approximately $2 \mathrm{~W}$ and average power at the sample was between $15 \mathrm{~mW}$ and $30 \mathrm{~mW}$.

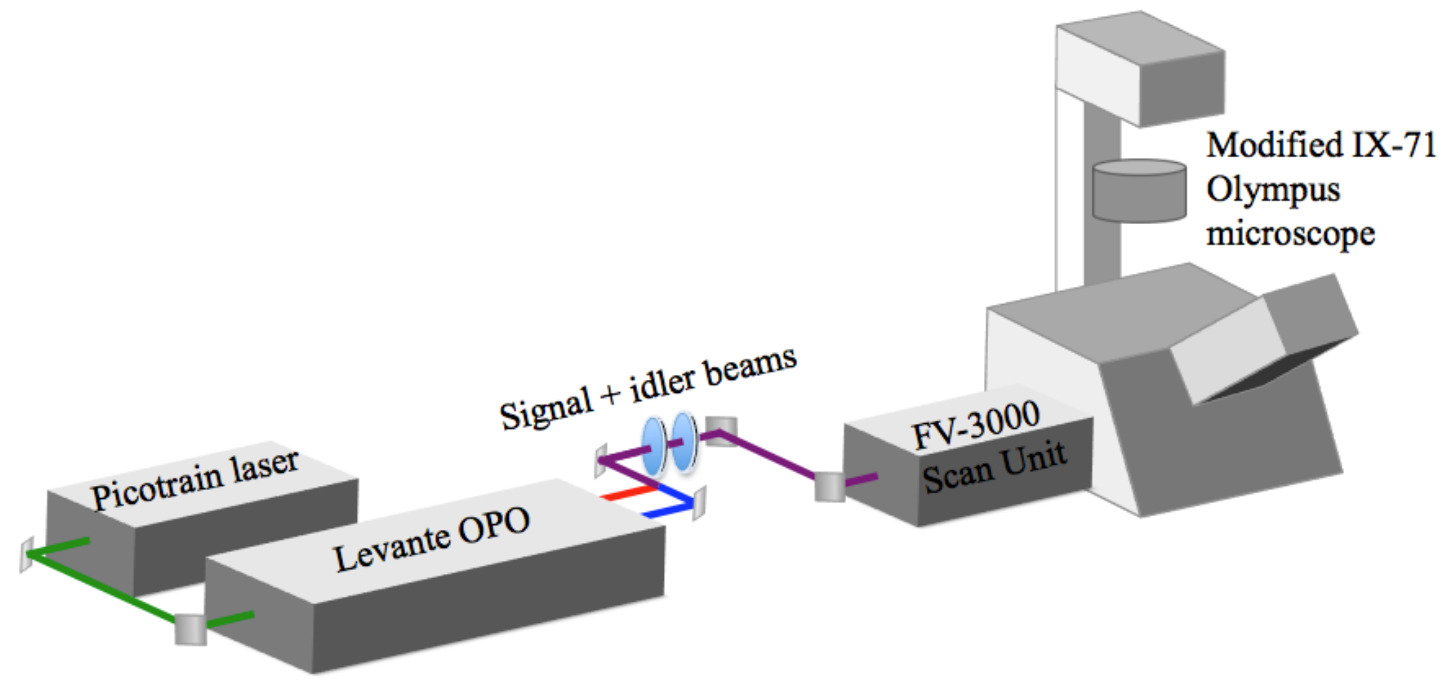

Figure 1. Spontaneous Raman scattering spectrum of dGCPQ nanoparticles, dried onto an aluminium substrate.

Imaging was performed using a modified inverted microscope and confocal laser scanner (IX71 and FV300, Olympus UK). A schematic of the optical setup is shown in Figure 1. To maximise IR transmission the standard galvanometer scanning mirrors were replaced with silver galvanometric mirrors, the tube lens was replaced with an $\mathrm{MgF} 2$ coated lens and the confocal dichroic was replaced by a silver mirror with high reflectivity throughout the visible and NIR (21010, Chroma Technologies, USA). A 60X, 1.2 NA water immersion objective (UPlanS Apo, Olympus UK) was used. 
The CARS signals were collected using the objective lens in the epi-direction. The epi-CARS signal was separated from the pump and Stokes beams by a long-wave pass dichroic mirror (z850rdc-xr, Chroma Technologies, USA) and directed onto a R3896 photomultiplier tube at the rear microscope port. The anti-Stokes signal was isolated by a single band-pass filter centered at $750 \mathrm{~nm}$ (HQ750/210, Chroma Technologies USA

\subsection{Sample preparation - deuterated GCPQ}

Acid degradation of glycol chitosan was performed using previously described techniques [ref]. A $50 \% \mathrm{v} / \mathrm{v}$ solution of ethanol and water was used to dissolve the degraded glycol chitosan before the addition of an ethanolic solution of deuterated palmitic acid (Palmitic acid D31,98 \% Cambridge Isotope Laboratories, Inc). Palmitoylation was facilitated by the addition of a coupling agent (4-Methylmorpholine, 4-[4,6-Dimeth- oxy-1,3,5-triazin-2-yl]-4-methylmorpholine chloride $>96.0 \%$, Sigma). Purified deuterated palmitoyl chitosan was then quaternised following Domard's method ${ }^{20}$ with some minor modifications ${ }^{1,21}$.

\subsection{Sample preparation - tissues}

All experiments were performed under a UK Home Office Animal License. CD-1 mice were fasted overnight and then administered dGCPQ formulations by oral gavage at the concentration of $1 \mathrm{mg} \mathrm{mL}^{-1}$. Mice were sacrificed at 4 hours and the kidneys were harvested. Tissues were either snap frozen or stored in neutral buffered formalin $(10 \% \mathrm{v} / \mathrm{v}, 15 \mathrm{~mL})$. Harvested kidneys were placed in a matrix (Zivic instruments) and cut in coronal slices of $0.5 \mathrm{~mm}$ thickness using cleaned razor blades. Each sample slice was placed onto a cleaned coverslip and placed within a window cut into four layers of Parafilm, which acted as a spacer, upon which a second coverslip was placed. A localised heat source was used to warm the edges of the Parafilm spacer to its melting point of $60{ }^{\circ} \mathrm{C}$, forming a watertight seal and preventing the samples from becoming desiccated.

\section{RESULTS AND DISCUSSION}

The spontaneous Raman scattering spectral profile of dGCPQ particles from $1900 \mathrm{~cm}^{-1}$ to $3200 \mathrm{~cm}^{-1}$ was acquired using Renishaw v.1.2 WiRE software and baseline subtracted using Origin 7, as shown in figure 2. Since the palmitoyl chain is the only region of the GCPQ molecule whose $\mathrm{CH}$ bonds are substituted for $\mathrm{CD}$ bonds, there is still a strong $\mathrm{CH}$-stretch exhibited from $\sim 2750 \mathrm{~cm}^{-1}-3050 \mathrm{~cm}^{-1}$. The CD stretch peak ranges from $\sim 2100 \mathrm{~cm}^{-1}-2250 \mathrm{~cm}^{-1}$ with the maximum peak height at $\sim 2105 \mathrm{~cm}^{-1}$. The corresponding CARS peak position for the CD stretch was located at $2100 \mathrm{~cm}^{-1}$.

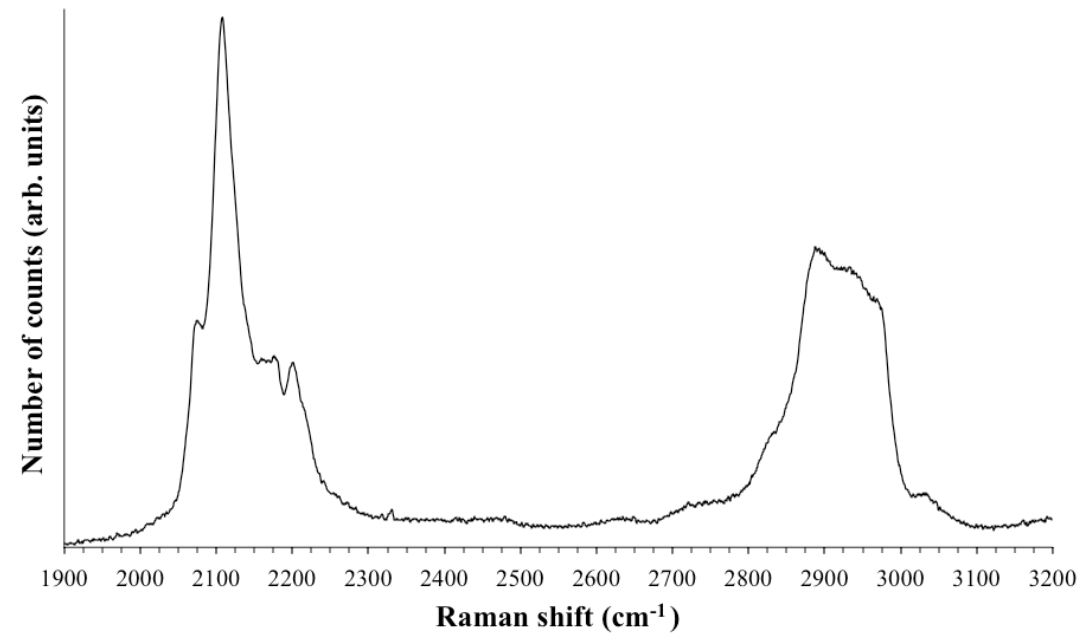

Figure 2. Spontaneous Raman scattering spectrum of dGCPQ nanoparticles, dried onto an aluminium substrate.

Successive epi-detected CARS image stacks were acquired of kidney samples using pump and Stokes wavelengths tuned to probe the resonance at $2100 \mathrm{~cm}^{-1}$ (to elicit contrast from the dGCPQ particles) and at $2848 \mathrm{~cm}^{-1}$ (to elicit contrast from endogenous $\mathrm{CH}$-rich structures in the sample, such as lipid droplets and cell membranes). The intensities of the pump and Stokes beams were monitored separately and each beam was attenuated to ensure the same power output when 
imaging using either $2100 \mathrm{~cm}^{-1}$ or $2845 \mathrm{~cm}^{-1}$ contrast. The total power at the sample was kept at $30 \mathrm{~mW}$, which was sufficiently low to prevent sample damage. Each x-y-z- image stack measured $171 \mu \mathrm{m}$ x $171 \mu \mathrm{m}$ x $150 \mu \mathrm{m}$ with z-step increments of $1 \mu \mathrm{m}$; each frame was acquired with a resolution of $256 \times 256$ pixels and a frame rate of 10 seconds. Using ImageJ software, the surface of the tissue within each sample stack was ascertained, and the signal intensity for the preceding 70 microns of tissue was determined for each channel (CD and $\mathrm{CH})$. The results for these experiments are shown in the graphs in figure 3.
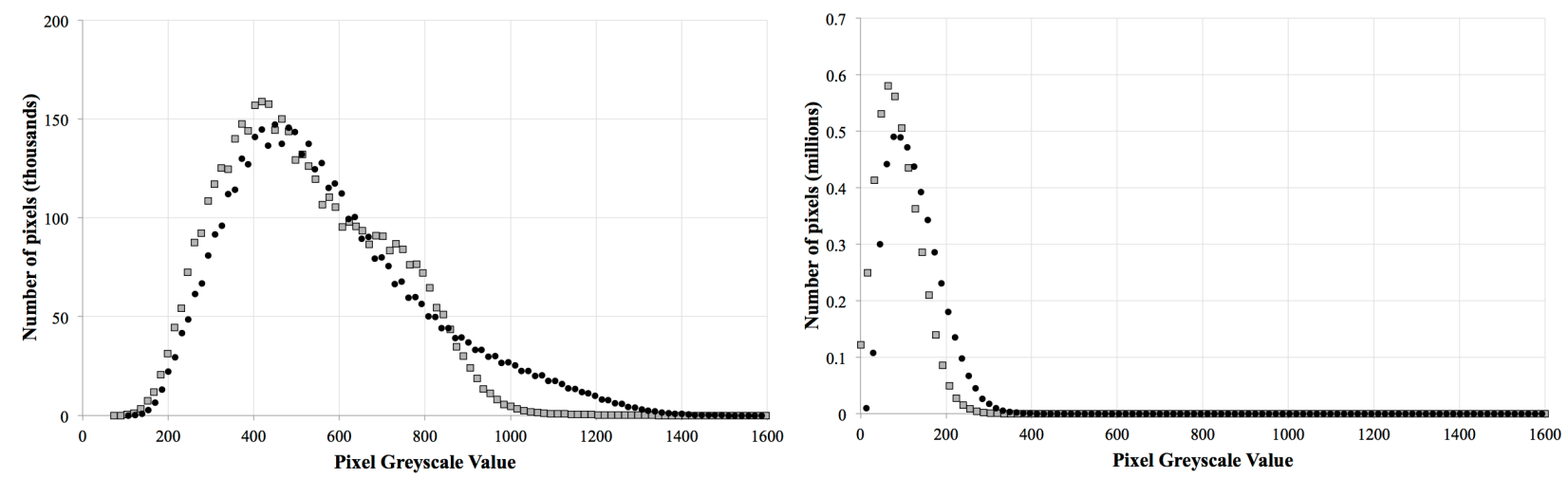

Figure 3. Epi-detected CARS signal intensity from fixed and unfixed kidney samples (black circle and grey square data points, respectively), obtained with pump and Stokes wavelengths tuned to probe the $\mathrm{CH}$-stretch at $2845 \mathrm{~cm}^{-1}$ (left) and CD-stretch at $2100 \mathrm{~cm}^{-1}$ (right).

The signal output from the microscope is 12-bit depth, thus the greyscale intensity values range from $0-4095$. Since only $<1 \%$ of the detected signal was above the greyscale value of 1600, the graphs in figure 2 have been cropped for ease of comparison. At the $\mathrm{CH}$ resonance, for the unfixed kidney tissue, the mean greyscale value was 528 with a standard deviation of 187 , whereas for the fixed kidney tissue this was 583 with a standard deviation of 236. At the CD resonance, for the unfixed kidney tissue the mean greyscale value was 96 with a standard deviation of 51, whereas for the fixed kidney tissue this was 133 with a standard deviation of 74 . For the CD resonance, deuterated GCPQ signal was typically found to exhibit pixel greyscale values of greater than 1600 .
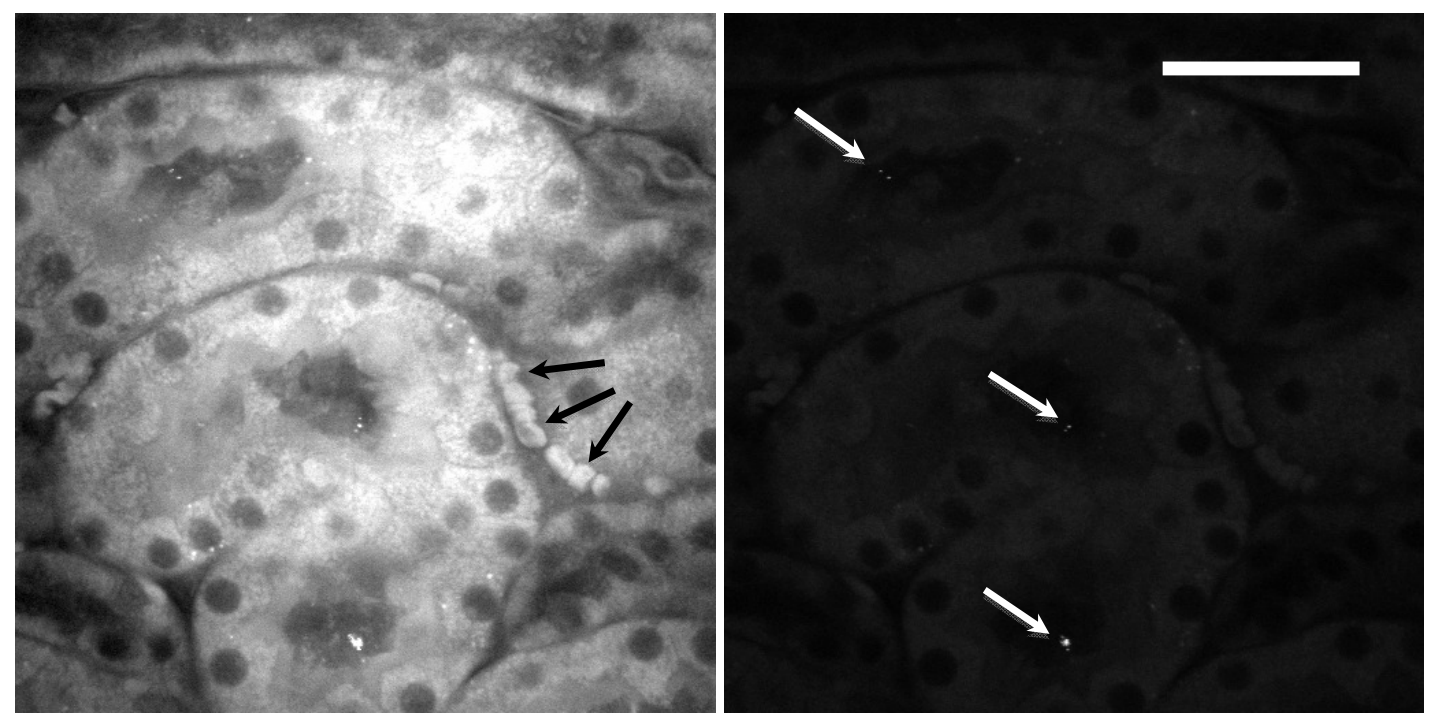

Figure 4. Epi-detected CARS images of a paraformaldehyde-fixed kidney sample, harvested after oral dosing with DGCPQ. Left $=\mathrm{CH}$ resonance, imaged using pump and Stokes wavelengths tuned to $2848 \mathrm{~cm}^{-1}$; right $=\mathrm{CD}$ resonance, imaged using pump and Stokes wavelengths tuned to $2100 \mathrm{~cm}^{-1}$. Black arrows indicate the position of red blood cells, white arrows indicate the position of dGCPQ particles. Scale bar is 50 microns. 
For fixed kidneys, when imaging at the $\mathrm{CH}$ stretch approximately $8.7 \%$ of the pixels exhibited CARS signal that was at a higher grey scale value than for snap-frozen kidneys, whereas when imaging at the CD stretch this figure increased to $25 \%$. Therefore, fixation-induced autofluorescence clearly has a greater impact on the detected CARS signal when imaging away from the $\mathrm{CH}$ resonance and further into the silent region. The increased background signal due to fixationinduced autofluorescence was not sufficiently great as to impede identification of resonant CD signal at $2100 \mathrm{~cm}^{-1}$, since the resonant signals yielded pixel greyscale values of greater than 1600 and the non-resonant background signal was below this threshold.
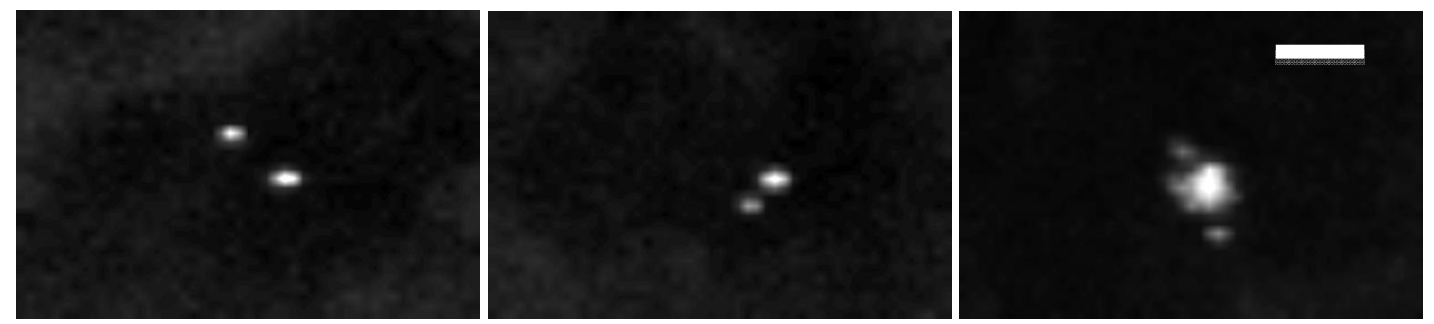

Figure 5. Higher magnification of the epi-detected CARS images of a the nanoparticles identified in the paraformaldehyde-fixed kidney sample shown in figure 4, harvested after oral dosing with DGCPQ nanoparticles. Scale bar is 10 microns.

Example images acquired from unfixed kidneys (figured $4-5$ ) and fixed kidneys (figures $6-7$ ). The nanoparticles identified at the $2100 \mathrm{~cm}^{-1}$ in figures 4 and 6 are shown in greater detail in figured 5 and 7 . The brightness and contrast of these images have not been adjusted. The nanoparticles were detected within the kidney tubule spaces, with no dGCPQ signal associated within cell bodies. This finding correlates well with previous work, which found that dGCPQ were excreted in the urine after oral administration ${ }^{2}$.
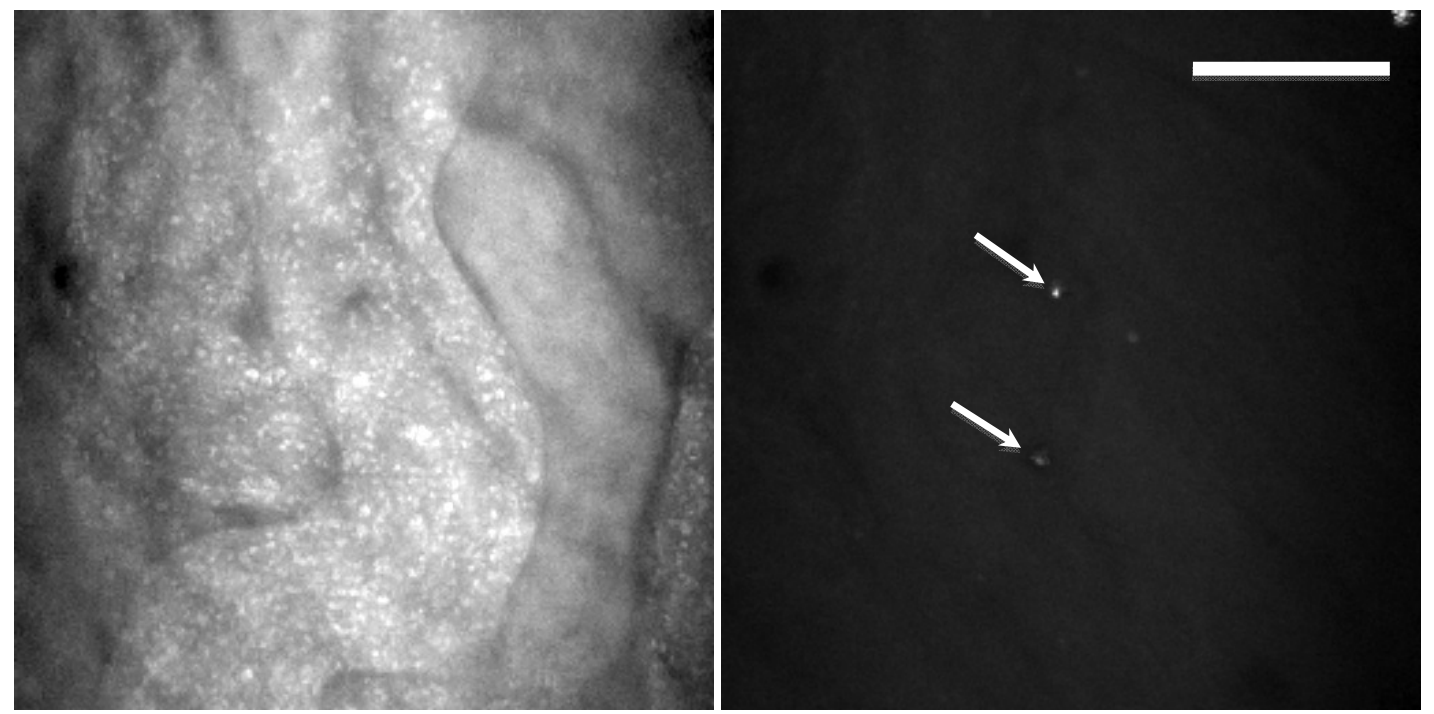

Figure 6. Epi-detected CARS images of an unfixed kidney sample, harvested after oral dosing with DGCPQ nanoparticles. Left = $\mathrm{CH}$ resonance, imaged using pump and Stokes wavelengths tuned to $2848 \mathrm{~cm}^{-1}$; right $=\mathrm{CD}$ resonance, imaged using pump and Stokes wavelengths tuned to $2100 \mathrm{~cm}^{-1}$. White arrows indicate the position of dGCPQ particles. Scale bar is 50 microns. 

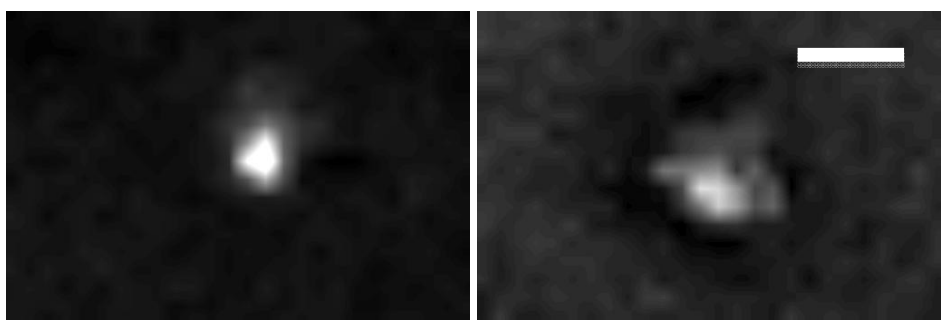

Figure 7. Epi-detected CARS images of an unfixed kidney sample, harvested after oral dosing with DGCPQ nanoparticles. Scale bar is 10 microns.

Since the pixel size is larger than the size of a single dGCPQ nanoparticle ( $660 \mathrm{~nm} \times 660 \mathrm{~nm}$ pixel size, c.f. average dGCPQ particle size of $\sim 200 \mathrm{~nm}$ ), a single pixel exhibiting dGCPQ signal does not necessarily correlate with a single nanoparticle. We discovered that the dGCPQ signal within the kidney tubules tended to be clustered in regions approximately $1-8$ microns in size, co-localised with strong $\mathrm{CH}$ signal.

\section{CONCLUSIONS}

We have shown that CARS microscopy is a robust tool that is capable of identifying deuterated GCPQ nanoparticles in both snap-frozen tissues and in tissues exhibiting fixative-induced autofluorescence. Although the non-resonant background levels of signal detected from fixed kidney sections was greater than for snap-frozen kidney sections, this signal level was several of orders of magnitude lower than that associated with the deuterated GCPQ resonant signal when imaging at the CD resonance at $2100 \mathrm{~cm}^{-1}$. Therefore, it was possible to simply and effectively screen out the nonresonant detected signal using image thresholding. However, our results indicate that when imaging nanoparticles exhibiting a weaker signal, the effects of fluorescence could have a greater negative impact on the detection sensitivity, therefore it is advisable to use snap frozen tissues where possible in such cases.

\section{REFERENCES}

[1] Qu, X., Khutoryanskiy, V. V., Stewart, A., Rahman, S., Papahadjopoulos-Sternberg, B., Dufès, C., McCarthy, D., Wilson, C. G., Lyons, R., et al., "Carbohydrate-based micelle clusters which enhance hydrophobic drug bioavailability by up to 1 order of magnitude," Biomacromolecules 7(12), 3452-3459, ACS Publications (2006).

[2] Lalatsa, A. ., Garrett, N. ., Ferrarelli, T. ., Moger, J. ., Schätzlein, A. .., Uchegbu, I., "Delivery of Peptides to the Blood and Brain after Oral Uptake of Quaternary Ammonium Palmitoyl Glycol Chitosan Nanoparticles," Mol. Pharm. (2012).

[3] Lalatsa, A., Schätzlein, A. G., Garrett, N. L., Moger, J., Briggs, M., Godfrey, L., Iannitelli, A., Freeman, J.., Uchegbu, I. F., "Chitosan amphiphile coating of peptide nanofibres reduces liver uptake and delivers the peptide to the brain on intravenous administration," J. Control. Release 197, 87-96 (2015).

[4] Lin, Y. H.., Salem, N., "Whole body distribution of deuterated linoleic and alpha-linolenic acids and their metabolites in the rat.," J. Lipid Res. 48(12), 2709-2724 (2007).

[5] Akira, K., Farrant, R. D., Lindon, J. C., Caddick, S. T., Nicholls, A. W.., Nicholson, J. K., "High-Field Deuterium Nuclear Magnetic Resonance Spectroscopic Monitoring of the Pharmacokinetics of Selectively Deuterated Benzoic Acid in Man,” Anal. Biochem. 221(2), 297-302 (1994).

[6] Bergner, G., Albert, C. R., Schiller, M., Bringmann, G., Schirmeister, T., Dietzek, B., Niebling, S., Schlücker, S.., Popp, J., "Quantitative detection of C-deuterated drugs by CARS microscopy and Raman microspectroscopy.," Analyst 2(1) (2011). 
[7] Van Manen, H.-J., Lenferink, A.., Otto, C., "Noninvasive imaging of protein metabolic labeling in single human cells using stable isotopes and Raman microscopy.," Anal. Chem. 80(24), 9576-9582 (2008).

[8] Evans, C. L.., Xie, X. S., "Coherent Anti-Stokes Raman Scattering Microscopy: Chemical Imaging for Biology and Medicine," Annu. Rev. Anal. Chem. 1, 883-909 (2008).

[9] Müller, M.., Zumbusch, A., "Coherent anti-Stokes Raman Scattering Microscopy.," Chemphyschem 8(15), 2156-2170 (2007).

[10] Nan, X. L., Cheng, J. X., Potma, E.., Xie, S. N., "Trafficking of lipid droplets in live steroidogenic cells studied with coherent anti-Stokes Raman scattering (CARS) microscopy,” Faseb J. 18(8), C51-C51 (2004).

[11] Garrett, N., Whiteman, M.., Moger, J., "Imaging the uptake of gold nanoshells in live cells using plasmon resonance enhanced four wave mixing microscopy," Opt. Express 19(18), 17563-17574 (2011).

[12] Moger, J., Johnston, B. D.., Tyler, C. R., "Imaging metal oxide nanoparticles in biological structures with CARS microscopy.," Opt. Express 16(5), 3408-3419 (2008).

[13] Xu, P., Gullotti, E., Tong, L., Highley, C. B., Errabelli, D. R., Hasan, T., Cheng, J.-X., Kohane, D. S.., Yeo, Y., "Intracellular drug delivery by poly(lactic-co-glycolic acid) nanoparticles, revisited.," Mol. Pharm. 6(1), 190201 (2009).

[14] Volkmer, A., Cheng, J. X.., Xie, X. S., "Vibrational imaging with high sensitivity via epidetected coherent antiStokes Raman scattering microscopy," Phys. Rev. Lett. 8702(2), 023901-1 - 023904-4 (2001).

[15] Evans, C. L.., Xie, X. S., "Coherent Anti-Stokes Raman Scattering Microscopy: Chemical Imaging for Biology and Medicine," Ann. Rev. Anal. Chem. 1(1), 883-909 (2008).

[16] Rodriguez, L. G., Lockett, S. J.., Holtom, G. R., "Coherent Anti-Stokes Raman Scattering Microscopy : A Biological Review," Cytometry 791, 779-791 (2006).

[17] Huang, S., Heikal, A. A.., Webb, W. W., "Two-photon fluorescence spectroscopy and microscopy of NAD(P)H and flavoprotein.,” Biophys. J. 82(5), 2811-2825 (2002).

[18] Ibrahim, M. N.., Sharif, S. E. A., "Synthesis, Characterization and Use of Schiff Bases as Fluorimetric Analytical Reagents," E-Journal of Chemistry 4(4), 531-535 (2007).

[19] Galli, R., Uckermann, O., Koch, E., Schackert, G., Kirsch, M.., Steiner, G., "Effects of tissue fixation on coherent anti-Stokes Raman scattering images of brain.,” J. Biomed. Opt. 19(7), 071402 (2014).

[20] Domard, A., Rinaudo, M.., Terrassin, C., "New method for the quaternization of chitosan," Int. J. Biol. Macromol. 8(2), 105-107 (1986).

[21] Siew, A., Le, H., Thiovolet, M., Gellert, P., Schatzlein, A.., Uchegbu, I., "Enhanced oral absorption of hydrophobic and hydrophilic drugs using quaternary ammonium palmitoyl glycol chitosan nanoparticles.," Mol. Pharm. 9(1), 14-28 (2012). 